\title{
Infinitesimal rigidity of a compact hyperbolic 4-orbifold with totally geodesic boundary
}

\author{
TARIK AOUGAB \\ PETER A STORM
}

\begin{abstract}
Kerckhoff and Storm conjectured that compact hyperbolic $n$-orbifolds with totally geodesic boundary are infinitesimally rigid when $n>3$. We verify this conjecture for a specific example based on the 4-dimensional hyperbolic 120-cell.
\end{abstract}

20F55, 20H10, 22E40

\section{Introduction}

Given a discrete subgroup $\Gamma$ of a semisimple Lie group $G$, mathematicians have long asked when $\Gamma$ can be deformed inside $G$. On one side lie the great rigidity theorems of Calabi, Weil, Mostow and Margulis, which roughly state that lattices in most semisimple Lie groups have no deformations. On the other side lie the beautiful deformation theories of discrete groups of $2 \times 2$ matrices, famous exceptions to the rigidity theorems. If $G$ is not represented by $2 \times 2$ matrices and $\Gamma$ is not a lattice, then nearly nothing is known about the possible deformations of $\Gamma$. Do reasonable geometric conditions exist that guarantee rigidity or flexibility?

As a first step in this direction, Kerckhoff and Storm studied deformations of a specific discrete subgroup of Isom $\left(\mathbb{H}^{4}\right)$, the isometry group of hyperbolic 4-space [5]. Of particular interest in this study were groups with Fuchsian ends that are not Fuchsian, meaning groups $\Gamma<\operatorname{Isom}\left(\mathbb{H}^{n}\right)$ whose convex hull is $n$-dimensional, has finite volume and has totally geodesic boundary. This led them to the following rigidity conjecture.

Conjecture 1.1 If a discrete group $\Gamma<\operatorname{Isom}\left(\mathbb{H}^{n}\right)$ has Fuchsian ends, is convex cocompact, is not Fuchsian and $n>3$, then the inclusion map of $\Gamma$ is infinitesimally rigid.

Drop any of the three conditions on $\Gamma$ and the conjecture becomes false. For any $n$, there are many convex cocompact discrete groups in $\operatorname{Isom}\left(\mathbb{H}^{n}\right)$ that are not rigid, for example if $\Gamma$ is a free group. For $n<4$ there exists a rich deformation theory applicable 
to discrete subgroups with Fuchsian ends. Even weakening the convex cocompact condition to geometric finiteness makes the conjecture false. A counterexample is presented in [5]. Nonetheless, it seems reasonable to expect that Conjecture 1.1 is true.

Before trying to prove the full conjecture, it seems prudent first to verify it in a nontrivial case. This is our goal here. More specifically, we will construct an explicit reflection group in $\operatorname{Isom}\left(\mathbb{H}^{4}\right)$ satisfying the hypotheses of Conjecture 1.1 and verify that it is infinitesimally rigid. The reflection group will be an infinite index subgroup of the lattice generated by reflections in the 3-cells of the right-angled hyperbolic 120-cell in $\mathbb{H}^{4}$. This discrete group was chosen carefully to make the computations as simple as possible. The group is described precisely in Section 3 and Section 4.

Acknowledgements The authors thank Daniel Allcock for his help with this research. This research was partially supported by NSF grant DMS-0741604. Storm also received support from the Roberta and Stanley Bogen Visiting Professorship at Hebrew University.

\section{Preliminaries}

This section is a brisk introduction to a few necessary facts from hyperbolic geometry. For a detailed introduction see, for example, Ratcliffe's book [6].

Recall that Minkowski $(n+1)$-space, denoted as $\mathbb{R}^{1, n}$ is a real $(n+1)$-dimensional vector space equipped with the nondegenerate symmetric bilinear form

$$
(\vec{x}, \vec{y}):=-x_{0} y_{0}+\sum_{i=1}^{n} x_{i} y_{i} .
$$

A vector with positive norm is a space-like vector, a vector of norm 0 is light-like and a vector with negative norm time-like. The hyperboloid model of hyperbolic $n$-space $\mathbb{H}^{n}$ is simply the set of vectors

$$
\left\{\vec{x} \in \mathbb{R}^{1, n} \mid(\vec{x}, \vec{x})=-1 \text { and } x_{0}>0\right\},
$$

with the metric induced by $\mathbb{R}^{1, n}$. Let $\mathrm{O}(1, n)$ be the group of real $(n+1) \times(n+1)$ matrices $A$ such that $A^{T} K A=K$, where $K$ is the $(n+1) \times(n+1)$ diagonal matrix with diagonal entries $\{-1,1,1, \ldots, 1\}$. In other words, $\mathrm{O}(1, n)$ is the group of linear transformations preserving the bilinear form $(\cdot, \cdot)$. The group $G_{n}:=\operatorname{Isom}\left(\mathbb{H}^{n}\right)$ of isometries of $\mathbb{H}^{n}$ is the open subgroup of $\mathrm{O}(1, n)$ given by matrices preserving the hyperboloid $\mathbb{H}^{n}$. Note that orientation reversing isometries are included here; $G_{n}$ has 
two connected components. Throughout the paper we will think of $G_{n}$ as this explicit group of matrices.

Consider a discrete finitely generated subgroup $\Gamma$ of $G_{n}$ with presentation

$$
\Gamma=\left\langle r_{1}, r_{2}, \ldots, r_{M} \mid w_{1}=w_{2}=\ldots=w_{N}=1\right\rangle,
$$

where each $w_{i}$ is a word in the generators $r_{j}$. (The letter $r$ stands for "reflection," which will soon be the focus.) To avoid degenerate cases, let us assume that $\Gamma$ is not virtually abelian and does not preserve a copy of $\mathbb{H}^{m}$ for $m<n$. If $\Gamma$ is torsion-free, then the quotient $\mathbb{H}^{n} / \Gamma$ will be a hyperbolic manifold. More generally, the quotient will be an orbifold. If the quotient space has finite volume then $\Gamma$ is called a lattice. Here we will be mainly interested in infinite volume quotient spaces, where a little more terminology is required.

Definition 2.1 The discrete group $\Gamma$ has Fuchsian ends and is not Fuchsian if there exists a closed $\Gamma$-invariant convex set $C_{\Gamma} \subseteq \mathbb{H}^{n}$ that is an $n$-manifold with nonempty totally geodesic boundary and such that the quotient $C_{\Gamma} / \Gamma$ has finite volume. Such a $\Gamma$ is convex cocompact if the quotient $C_{\Gamma} / \Gamma$ is compact.

We will not need it here, but $\Gamma<G_{n}$ is Fuchsian if it is conjugate into the subgroup $G_{n-1}$. In the Fuchsian case the corresponding $C_{\Gamma}$ would be a manifold of dimension at most $n-1$.

If $\Gamma$ has Fuchsian ends and is not Fuchsian, then $\partial C_{\Gamma}$ consists of an infinite number of disjoint totally geodesic hyperplanes, each isometric to $\mathbb{H}^{n-1}$. The quotient $C_{\Gamma} / \Gamma$ is a finite volume orbifold with totally geodesic boundary. For the reader familiar with convex cores, we note that $C_{\Gamma}$ is the convex core of $\Gamma$.

Consider the representation variety $\operatorname{Hom}\left(\Gamma, G_{n}\right)$. The slightly larger representation space $\operatorname{Hom}(\Gamma, \mathrm{O}(1, n))$ can be represented explicitly as the zero set of a collection of real polynomials as follows. Recall $\Gamma$ is generated by $\left\{r_{1}, r_{2}, \ldots, r_{M}\right\}$. A homomorphism $\rho: \Gamma \rightarrow \mathrm{O}(1, n)$ is determined uniquely by the $M$ matrices $\rho\left(r_{j}\right)$ of $\mathrm{O}(1, n)$, which can be thought of as a point in a real vector space $V$ of dimension $M \cdot(n+1)^{2}$. The $\rho\left(r_{j}\right)$ must lie in $\mathrm{O}(1, n)$, meaning for each $j$ the $(n+1)^{2}$ polynomials $\rho\left(r_{j}\right)^{T} K \rho\left(r_{j}\right)=K$ are satisfied. Each of the $N$ relations $w_{i}$ of $\Gamma$ becomes a system of $(n+1)^{2}$ polynomial equations in the coordinates of $V$. The variety $\operatorname{Hom}(\Gamma, \mathrm{O}(1, n)) \subset V$ is precisely the set of points where these $(M+N) \cdot(n+1)^{2}$ polynomial equations $\left\{P_{\alpha}\right\}$ are satisfied. Finally, we are interested in the subset $\operatorname{Hom}\left(\Gamma, G_{n}\right) \subset \operatorname{Hom}(\Gamma, \mathrm{O}(1, n))$. It is the set of connected components where the upper-left matrix entry of each $\rho\left(r_{j}\right)$ is positive. (This entry cannot be zero for a matrix in $\mathrm{O}(1,4)$.) 
The group $G_{n}$ acts algebraically on $\operatorname{Hom}\left(\Gamma, G_{n}\right)$ by conjugation as follows:

$$
(g \cdot \rho)(\gamma):=g \rho(\gamma) g^{-1} .
$$

The inclusion map $\Gamma \rightarrow G_{n}$ is a point $\iota \in \operatorname{Hom}\left(\Gamma, G_{n}\right)$. In general, analyzing the orbits of this $G_{n}$-action is complicated. However, using our assumptions that $\Gamma$ is not virtually abelian and does not preserve a lower dimensional hyperbolic space, it is easy to show that the orbit $G_{n} \cdot \iota \subseteq \operatorname{Hom}\left(\Gamma, G_{n}\right)$ is a manifold of dimension equal to that of $G_{n}$; see Goldman [4].

A homomorphism $\rho \in \operatorname{Hom}\left(\Gamma, G_{n}\right)$ is locally rigid if it has an open neighborhood contained inside the orbit $G_{n} \cdot \rho$; in other words all nearby representations are obtained by conjugation. The infinitesimal analogue of this notion is useful. An infinitesimal deformation of $\rho$ is a tangent vector $p^{\prime}(0) \in T_{\rho} V$ to a smooth path $p:(-\varepsilon, \varepsilon) \rightarrow V$ such that

$$
\left.\frac{d}{d t}\right|_{0} P_{\alpha}(p(t))=0
$$

for the $(M+N) \cdot(n+1)^{2}$ polynomial equations $\left\{P_{\alpha}\right\}$ defining $\operatorname{Hom}(\Gamma, \mathrm{O}(1, n))$ (and locally defining $\operatorname{Hom}\left(\Gamma, G_{n}\right)$ ). This slightly odd definition is necessitated by the possibility that $\operatorname{Hom}\left(\Gamma, G_{n}\right)$ is singular at $\rho$. Similarly, an infinitesimal conjugation of $\rho$ is a tangent vector $\vec{v} \in T_{\rho} V$ such that

$$
\vec{v}=\left.\frac{d}{d t}\right|_{0}(g(t) \cdot \rho)
$$

for some smooth path $g:(-\varepsilon, \varepsilon) \rightarrow G_{n}$ satisfying $g(0)=\mathrm{Id}$. We say $\rho$ is infinitesimally rigid if every infinitesimal deformation is an infinitesimal conjugation. An infinitesimally rigid homomorphism is also locally rigid [10]. By the above discussion, to show that the inclusion map ८ of $G_{n}$ is infinitesimally rigid, it suffices to show that the linear subspace of infinitesimal deformations has dimension equal to that of $G_{n}$.

When $\Gamma<G_{n}$ is a lattice, we have the famous rigidity theorem of Calabi, Weil and Garland.

Theorem $2.2[1 ; 10 ; 3]$ If $\Gamma<\operatorname{Isom}\left(\mathbb{H}^{n}\right)$ is a lattice and $n>3$ then the inclusion map of $\Gamma$ is infinitesimally rigid.

For discrete groups that are not lattices, Theorem 2.2 is false, but it is interesting to study whether or not a similar rigidity theorem might hold for other natural families of discrete groups. Looking for infinite covolume groups that are as "close" to lattices as possible, Kerckhoff and Storm were led to consider discrete groups with Fuchsian ends that are not Fuchsian. In $\mathbb{H}^{3}$, groups with Fuchsian ends are not rigid and have a beautiful deformation theory investigated by many people (see Thurston [8]). In higher 
dimensions, simple dimension counts suggest non-Fuchsian groups with Fuchsian ends are rigid. These dimension counts are, of course, not rigorous. Nonetheless, Kerckhoff and Storm were led to the following conjecture, stated first in the introduction.

Conjecture 1.1 If a discrete group $\Gamma<\operatorname{Isom}\left(\mathbb{H}^{n}\right)$ has Fuchsian ends, is convex cocompact, is not Fuchsian and $n>3$, then the inclusion map of $\Gamma$ is infinitesimally rigid.

Roughly speaking, a group $\Gamma$ satisfying the hypotheses of Conjecture 1.1 is similar to a lattice in $G_{n}$. Its covolume is infinite, but $\Gamma$ is nonetheless intuitively quite large inside $G_{n}$. Note that Conjecture 1.1 is false without the assumption that $\Gamma$ is convex cocompact. A counterexample was studied in [5]. As a first step toward proving this conjecture, our goal is to verify it in a specific 4-dimensional example. In search of an example where the computations are as simple as possible, the first place to look is among hyperbolic reflection groups, which we now introduce briefly. For more information the authors recommend Vinberg and Shvartsman [9].

A reflection isometry in $G_{4}=\operatorname{Isom}\left(\mathbb{H}^{4}\right)$ is given by a matrix in $\mathrm{O}(1,4)$ with a 4dimensional eigenspace of eigenvalue 1 and an eigenvalue -1 whose corresponding eigenvector is space-like. It fixes a codimension 1 totally geodesic hyperplane of $\mathbb{H}^{4}$ given by the intersection of its +1 -eigenspace with $\mathbb{H}^{4}$. The orthogonal complement of the fixed hyperplane is reflected across the hyperplane by the isometry. The reflection isometry is determined uniquely by the hyperplane and vice versa.

Consider the interesting special case where $\Gamma<G_{4}$ is a group generated by $M$ reflections $r_{1}, r_{2}, \ldots, r_{M}$ with corresponding hyperplanes $H_{1}, H_{2}, \ldots, H_{M}$, and the hyperplanes bound a convex (possibly infinite volume) region $P$ of $\mathbb{H}^{4}$ known as a fundamental domain. Moreover, assume the dihedral angles of $P$ are all $\pi / 2$. In this case the presentation of $\Gamma$ takes the following nice form [9]:

$$
\Gamma=\left\langle r_{1}, r_{2}, \ldots, r_{M} \mid\left(r_{i} r_{j}\right)^{m_{i j}}=r_{i}^{2}=1\right\rangle,
$$

where for each pair $1 \leq i<j \leq M, m_{i j}$ is either 2 or infinity. The "relation" $\left(r_{i} r_{j}\right)^{\infty}=1$ indicates the product $r_{i} r_{j}$ has infinite order. The product $r_{i} r_{j}$ of reflections has order 2 when the hyperplanes $H_{i}$ and $H_{j}$ intersect at angle $\pi / 2$. This is best seen by thinking about the picture in the Euclidean plane of the product of two reflections. Otherwise the product has infinite order, or $m_{i j}=\infty$. (Here we are not allowing intersections at other angles.)

We will be considering the representation variety $\operatorname{Hom}\left(\Gamma, G_{4}\right) \subset \mathbb{R}^{25 M}$ for groups $\Gamma$ of the above form. 
The following lemma shows that near the inclusion map in $\operatorname{Hom}\left(\Gamma, G_{4}\right)$ any representation has the property that it maps the generators $r_{i}$ to reflection isometries of $G_{4}$.

Lemma 2.3 Let $A \in G_{n}$ be a reflection isometry. Then $A$ has an open neighborhood $U \subset G_{n}$ such that if $B \in U$ and $B^{2}=\mathrm{Id}$, then $B$ is a reflection isometry.

Proof Recall that any involution can be diagonalized over $\mathbb{C}$ such that the diagonal entries are all \pm 1 . Recall also that a reflection must have exactly one eigenvalue equal to -1 . We can define the open neighborhood $U$ of $A$ to be all matrices in $G_{n}$ having $(n-1)$ positive eigenvalues (not necessarily distinct) and 1 negative eigenvalue. Then any matrix in $U$ that is an involution will necessarily be a nontrivial isometry which fixes a hyperplane of codimension 1 , in other words, a reflection.

Corollary 2.4 There exists an open neighborhood $U \subset \operatorname{Hom}\left(\Gamma, G_{4}\right) \subset \mathbb{R}^{25 M}$ of the inclusion map such that if $\rho \in U$, then $\rho\left(r_{i}\right)$ is a reflection for all $i$.

The representation variety $\operatorname{Hom}\left(\Gamma, G_{4}\right)$ sits naturally as (connected components of) a subvariety of $\mathbb{R}^{25 M}$, but by exploiting the fact that locally all the generators are reflections, it is possible to reduce the dimension considerably. To a reflection isometry $r$ with fixed hyperplane $H$ we can associate the 1 -dimensional subspace of $\mathbb{R}^{1,4}$ given by vectors Minkowski orthogonal to $H$. This subspace is simply the -1 -eigenspace of the matrix corresponding to $r$, and it will consist of space-like vectors. In reverse, choosing a space-like vector $\vec{n}$ determines a codimension 1 hyperplane $\vec{n}^{\perp} \cap \mathbb{H}^{4}$, which in turn determines a reflection isometry. Letting $\mathcal{S} \subset \mathbb{R}^{1,4}$ denote the set of space-like vectors, this process defines a map

$$
\mu: \mathcal{S}^{M} \rightarrow G_{4}^{M} \subset \mathbb{R}^{25 M}
$$

with image equal to the set of $M$-tuples of reflection isometries.

Moreover, the geometry of the fundamental domain $P$ can be read from the normal vectors. Specifically, let $\vec{n}_{1}$ and $\vec{n}_{2}$ be space-like in $\mathbb{R}^{1,4}$, and

$$
H_{i}:=\left\{\vec{v} \in \mathbb{R}^{1,4} \mid\left(\vec{v}, \vec{n}_{i}\right)=0\right\} .
$$

If $H_{1} \cap H_{2} \cap \mathbb{H}^{4} \neq \varnothing$ and $H_{1}$ and $H_{2}$ intersect at angle $\theta$, then

$$
\frac{\left(\vec{n}_{1}, \vec{n}_{2}\right)}{\sqrt{\left(\vec{n}_{1}, \vec{n}_{1}\right)\left(\vec{n}_{2}, \vec{n}_{2}\right)}}=-\cos (\theta) \text {. }
$$


We will only consider examples where $\theta$ is always $\pi / 2$, in which case the above simplifies to

$$
\left(\vec{n}_{1}, \vec{n}_{2}\right)=0 .
$$

If on the other hand $H_{1} \cap H_{2} \cap \mathbb{H}^{4}=\varnothing$ and the shortest hyperbolic geodesic from $H_{1} \cap \mathbb{H}^{4}$ to $H_{2} \cap \mathbb{H}^{4}$ has length $\ell$ then

$$
\frac{\left(\vec{n}_{1}, \vec{n}_{2}\right)}{\sqrt{\left(\vec{n}_{1}, \vec{n}_{1}\right)\left(\vec{n}_{2}, \vec{n}_{2}\right)}}=-\cosh (\ell) .
$$

Choose a representation $\rho \in \operatorname{Hom}\left(\Gamma, G_{4}\right)$ sending each generator $r_{i}$ to a reflection. In the above manner, we replace every reflection matrix $\rho\left(r_{i}\right)$ by a corresponding normal vector $\vec{n}_{i}$. We now have $M$ Minkowski 5-vectors, instead of $M 5 \times 5$ matrices. The relations of $\Gamma$ written in terms of normal vectors all have the form

$$
\left(\vec{n}_{i}, \vec{n}_{j}\right)=0 \quad \text { when } m_{i j}=2 .
$$

Let $U \subset \operatorname{Hom}\left(\Gamma, G_{4}\right)$ be the open neighborhood of the inclusion map of Corollary 2.4. It is clear that, restricted to $\mu^{-1}(U), \mu$ is a submersion with fibers given by scaling the normal vectors $\vec{n}_{i}$. Given a vector $v=\left(\dot{\vec{n}}_{i}\right)_{i=1}^{M}$ tangent to the point $\left(\vec{n}_{i}\right) \in \mu^{-1}(\rho)$, $\mu_{*} v \in T_{\rho} \mathbb{R}^{25 M}$ is an infinitesimal deformation of $\rho$ if and only if $v$ satisfies the following derivatives of Equation (2):

$$
\left(\dot{\vec{n}}_{i}, \vec{n}_{j}\right)+\left(\vec{n}_{i}, \dot{\vec{n}}_{j}\right)=0 \quad \text { when } m_{i j}=2 .
$$

Any solution to this system of polynomials will now be a vector $\left(\dot{\vec{n}}_{i}\right)$ in $\mathbb{R}^{5 M}$ instead of $\mathbb{R}^{25 M}$. Note that we do not need to include each generator's involution relation. This interpretation clearly has an immense computational advantage over working with the matrices directly.

Finally, to show $\rho$ is infinitesimally rigid in $\operatorname{Hom}\left(\Gamma, G_{4}\right)$ it suffices to show that the subspace of vectors $\left(\dot{\vec{n}}_{i}\right) \subset \mathbb{R}^{5 M}$ satisfying system (3) has dimension equal to $10+M$, where $10=\operatorname{dim}\left(G_{4}\right)$ dimensions come from infinitesimal conjugations by $G_{4}$, and $M$ dimensions correspond to scaling the normal vectors, one for each generator of $\Gamma$.

\section{The 120-cell}

Here we will outline some of the basic properties of the hyperbolic $120-$ cell. We will use the word face to indicate a 2-cell and wall to indicate a 3-cell. The 120-cell is a regular convex polytope formed by 120 dodecahedral walls, where regular means that its symmetry group acts transitively on the set of flags. (A flag of the 120-cell is a quadruple consisting of 1 point, 1 edge containing the point, 1 pentagonal face 
containing the edge and 1 dodecahedral wall containing the face.) The $120-$ cell has 600 vertices and is dual to the 600 -cell formed by 600 icosahedra.

The 120 -cell can be embedded into $\mathbb{H}^{4}$ such that the resulting convex hyperbolic polytope is compact, regular, and all intersecting dodecahedra hit at right angles. The quickest way to describe this polytope is by giving a list of 120 space-like vectors that are Minkowski-normal to the 120 walls. For completeness, we list the 120 normal vectors in $\mathbb{R}^{1,4}$, using the golden ratio $\tau=(1+\sqrt{5}) / 2[2]$ :

(i) 8 vectors obtained by permuting the last 4 coordinates of $(\sqrt{2 \tau}, \pm 2,0,0,0)$

(ii) 16 vectors of the form $(\sqrt{2 \tau}, \pm 1, \pm 1, \pm 1, \pm 1)$

(iii) 96 even permutations in the last 4 coordinates of $\left(\sqrt{2 \tau}, \pm \tau, \pm 1, \pm \tau^{-1}, 0\right)$

Consider the set $\mathcal{C}$ of walls of the 120 -cell given by the 24 space-like vectors of items (i) and (ii) above. Interestingly, these 24 vectors determine 24 hyperplanes of $\mathbb{H}^{4}$ which bound a regular (hyperideal) hyperbolic 24-cell, which is a polytope with 24 octahedral walls. This set $\mathcal{C}$ will play an important role here. Using Equation (1) one can compute directly that the walls of $\mathcal{C}$ are pairwise disjoint in $\mathbb{H}^{4}$.

Lemma 3.1 There does not exist a set of 25 pairwise disjoint walls in the 120 -cell.

Proof Suppose we remove 24 pairwise disjoint walls from the 120-cell. For each wall that we remove, we place a marker on any wall which was adjacent to the removed wall. Each wall of the 120 -cell is adjacent to 12 other walls; therefore after removing 24 walls we have placed $24 \times 12=288$ markers on the remaining 96 walls or an average of 3 markers per wall that remains. The claim is that every remaining wall has exactly 3 markers. If this is indeed true, then removing a 25-th disjoint wall would be impossible because any wall we removed would be adjacent to 3 of the walls in the original set of 24 .

Suppose one of the walls had only 2 markers. Then there is at least one other wall with 4 markers, implying the intersection of a certain set of 4 pairwise disjoint walls with this wall of 4 markers is a set of 4 pairwise disjoint pentagonal faces of a dodecahedron. This is impossible because the maximum number of pairwise disjoint faces of a dodecahedron is 3 . Therefore no wall has 2 or fewer markers implying every wall has 3 .

The above proof was explained to us by Allcock. 


\section{The main theorem}

We are now ready to discuss the main result.

Theorem 4.1 Let $P \subset \mathbb{H}^{4}$ be the hyperbolic regular right-angled 120-cell. Let $\mathcal{C}$ be the set of 24 pairwise disjoint walls of $P$ given in Section 3. Let $\Gamma<G_{4}=$ Isom $\left(\mathbb{H}^{4}\right)<\mathrm{O}(1,4)$ be the discrete infinite covolume group generated by the reflections in the 96 walls of $P$ that are not in $\mathcal{C}$. Then the inclusion map $\Gamma \rightarrow G_{4}$ is infinitesimally rigid in the representation variety $\operatorname{Hom}\left(\Gamma, G_{4}\right)$.

Before beginning the proof let us discuss the connection to Conjecture 1.1.

Proposition 4.2 Let $\Gamma<G_{4}$ be the discrete group of Theorem 4.1. Then $\Gamma$ has Fuchsian ends and the quotient $C_{\Gamma} / \Gamma$ is isometric to the $120-$ cell $P$. In particular, $\Gamma$ is convex cocompact and not Fuchsian.

Proof Consider the collection of 24 dodecahedral walls $\mathcal{C} \subset \mathbb{H}^{4}$ and its orbit $\Gamma \cdot \mathcal{C}$. Using the facts that all the dihedral angles of $P$ are $\pi / 2$, and all the walls of $\mathcal{C}$ are pairwise disjoint, it follows that any intersecting translates of $\mathcal{C}$ in the orbit $\Gamma \cdot \mathcal{C}$ glue together smoothly. In particular, the entire orbit is a disjoint union of totally geodesic hyperplanes. Let $C_{\Gamma} \subset \mathbb{H}^{4}$ be the $\Gamma$-invariant convex subset bounded by $\Gamma \cdot \mathcal{C}$. This shows that $\Gamma$ has Fuchsian ends. It is clear that the quotient $C_{\Gamma} / \Gamma$ is isometric to $P$.

Theorem 4.1 and Proposition 4.2 imply Conjecture 1.1 is true for $\Gamma$.

Corollary 4.3 The discrete subgroup $\Gamma<G_{4}$ has Fuchsian ends, is convex cocompact and infinitesimally rigid, as predicted by Conjecture 1.1.

To prove Theorem 4.1, we begin with $965 \times 5$ reflection matrices in $G_{4}<\mathrm{O}(1,4)$, the image of the generators of $\Gamma$ under the inclusion map. Replace each such reflection matrix with one of its (space-like) eigenvectors $\vec{n}_{i}$ corresponding to eigenvalue -1 . (All possible choices are colinear.) The complete list of 96 vectors $\vec{n}_{i}$ is the 96 even permutations in the last 4 coordinates of $\left(\sqrt{2 \tau}, \pm \tau, \pm 1, \pm \tau^{-1}, 0\right)$, where $\tau$ is the golden ratio. Pairs $\vec{n}_{i}$ and $\vec{n}_{j}$ corresponding to orthogonal walls of $P$ satisfy the relation

$$
\left(\vec{n}_{i}, \vec{n}_{j}\right)=0 .
$$

Let $W \subset T_{\vec{n}} \mathbb{R}^{480}$ be the linear space of infinitesimal deformations. Suppose we are given an infinitesimal deformation

$$
\left(\dot{\vec{n}}_{i}\right) \in W \subset T_{\vec{n}} \mathbb{R}^{480} \cong \mathbb{R}^{480}
$$


of the 96 space-like vectors which maps via $\mu_{*}$ to an infinitesimal deformation of $\Gamma$ in $G_{4}$. Then the following linear equation must hold for all pairs $i, j$ corresponding to intersecting orthogonal walls of $P$ :

$$
\left(\dot{\vec{n}}_{i}, \vec{n}_{j}\right)+\left(\vec{n}_{i}, \dot{\vec{n}}_{j}\right)=0 .
$$

Keep in mind that the 24 walls of $\mathcal{C}$ have been removed from $P$. There are 432 equations of this type, corresponding to the 432 remaining faces of $P$. To prove that $\Gamma$ is infinitesimally rigid it suffices to show the solution space $W$ has $10+96$ dimensions, where 10 comes from the action of $G_{4}$ by conjugation, and 96 comes from scaling each of the 96 space-like vectors $\vec{n}_{i}$. These 96 scaling dimensions are killed by $\mu_{*}$. It is easy to see the solution space $W$ has at least 106 dimensions. It remains to show it has at most 106 dimensions.

Enumerate the polynomials $\left\{p_{1}, p_{2}, \ldots, p_{432}\right\}$ defining $W$. Each $p_{i}$ is a polynomial in the variables $\left(v_{1}, v_{2}, \ldots, v_{480}\right)$ where

$$
\dot{\vec{n}}_{i}=\left(v_{5(i-1)+1}, v_{5(i-1)+2}, v_{5(i-1)+3}, v_{5(i-1)+4}, v_{5 i}\right) \text {. }
$$

Define the matrix $A_{\text {alg }}$ of algebraic numbers

$$
\left(A_{\mathrm{alg}}\right)_{i j}:=\frac{d}{d v_{j}}\left(p_{i}\left(v_{1}, v_{2}, \ldots, v_{480}\right)\right) .
$$

Each entry $\left(A_{\text {alg }}\right)_{i j}$ is a number because each polynomial $p_{i}$ is linear in the variables $v_{j}$. $A_{\text {alg }}$ has 432 rows and 480 columns. The goal is to show its kernel has dimension at most 106 . We do this by showing the rank is at least 374 .

We bounded the rank from below using the computer algebra system Mathematica. (For computer related information see Remark 4.4 at the end of the paper.) In order to ensure Mathematica can correctly and quickly bound the rank, we replace every algebraic number entry of $A_{\text {alg }}$ with a square matrix of rational numbers. The number field $\mathbb{Q}(\alpha)$ contains every entry of $A_{\text {alg }}$ when $\alpha=\sqrt{1+\sqrt{5}}$. We choose the basis

$$
\left\{1, \alpha, \alpha^{2}, \alpha^{3}\right\}
$$

for $\mathbb{Q}(\alpha)$ as a vector space over $\mathbb{Q}$. Then each entry of $A_{\text {alg }}$ is replaced by a $4 \times 4$ rational matrix representing its action by left multiplication on $\mathbb{Q}(\alpha)$ with respect to this basis. For example, the number $\frac{1}{2}(-1-\sqrt{5})$ is replaced by the $4 \times 4$ matrix:

$$
\left(\begin{array}{cccc}
0 & 0 & -2 & 0 \\
0 & 0 & 0 & -2 \\
-\frac{1}{2} & 0 & -1 & 0 \\
0 & -\frac{1}{2} & 0 & -1
\end{array}\right)
$$


Once we have the rational $4 \times 4$ matrix replacements for each entry of $A_{\text {alg }}$, it becomes a $432 \times 480$ matrix of $4 \times 4$ matrices. By ignoring the structure of the $4 \times 4$ matrices, we can think of it as a $1728 \times 1920$ matrix $A_{\mathbb{Q}}$ of rational numbers. It is easy to see that the kernel of $A_{\mathbb{Q}}$ has dimension at least 4 times that of $A_{\text {alg. }}$ (If $u$ is in the kernel of $A_{\text {alg }}$ then $\left\{u, \alpha u, \alpha^{2} u, \alpha^{3} u\right\}$ will be in the kernel of $A_{\mathbb{Q}}$ and linearly independent over $\mathbb{Q}$.) Therefore the rank of $A_{\mathbb{Q}}$ is at most 4 times the rank of $A_{\text {alg }}$. To prove the infinitesimal rigidity of $\Gamma$, it suffices to show that the rank of $A_{\mathbb{Q}}$ is at least $4 \cdot 374=1496$.

In order to further simplify the computation, multiply each row of $A_{\mathbb{Q}}$ by the least common multiple of the denominators of the rational numbers in that row. This gives us the integer matrix $A_{\mathbb{Z}}$ with the same rank as $A_{\mathbb{Q}}$. With some effort on a desktop computer (in 2008), Mathematica was able to determine that the rank of this matrix is 1496, the desired result. Therefore $A_{\text {alg }}$ has a kernel of dimension at most 106 . This proves that the inclusion map of the discrete group $\Gamma<G_{4}$ is infinitesimally rigid in the representation variety $\operatorname{Hom}\left(\Gamma, G_{4}\right)$.

The rank calculation for $A_{\mathbb{Z}}$ took approximately 13 seconds. However, some machines may have more difficulty. The calculation can be simplified by reducing $A_{\mathbb{Z}}$ modulo a suitable prime $p$, obtaining matrix $A_{p}$. Diagonalization is much easier in the finite field of order $p$, and $\operatorname{rank}\left(A_{p}\right) \leq \operatorname{rank}\left(A_{\mathbb{Z}}\right)$. Therefore, simply reduce $A_{\mathbb{Z}}$ modulo primes until one is found satisfying $\operatorname{rank}\left(A_{p}\right)=1496$. The prime 3 suffices, producing a rank of 1496 in approximately 2 seconds.

Remark 4.4 The main calculations were performed in 2008 using Wolfram's Mathematica versions 6 and 7 on a MacPro running 64-bit Linux. They were checked using both Maple 11 and Sage 3.2.3. Detailed descriptions of the above computations, including the relevant computer code, are available on the website of the second author [7].

\section{References}

[1] E Calabi, On compact, Riemannian manifolds with constant curvature. I, from: "Proc. Sympos. Pure Math., Vol. III", Amer. Math. Soc. (1961) 155-180 MR0133787

[2] H S M Coxeter, Introduction to geometry, Wiley Classics Library, Wiley, New York (1989) MR990644 Reprint of the 1969 edition

[3] H Garland, On deformations of discrete groups in the noncompact case, from: "Algebraic Groups and Discontinuous Subgroups (Proc. Sympos. Pure Math., Boulder, Colo., 1965)”, Amer. Math. Soc. (1966) 405-412 MR0206166 
[4] W M Goldman, The symplectic nature of fundamental groups of surfaces, Adv. in Math. 54 (1984) 200-225 MR762512

[5] SP Kerckhoff, PA Storm, From the 24-cell to the cuboctahedron arXiv: 0805.4537

[6] J G Ratcliffe, Hyperbolic manifolds, from: "Handbook of geometric topology", (R J Daverman, R B Sher, editors), North-Holland, Amsterdam (2002) 899-920 MR1886683

[7] PA Storm, Web site Available at http://www.sas.upenn.edu/ pstorm/ index.html

[8] W P Thurston, The geometry and topology of three-manifolds, Princeton Univ. Math. Dept. Lecture Notes (1979) Available at http://msri.org/publications/books/ gt $3 \mathrm{~m} /$

[9] ̇̀ B Vinberg, O V Shvartsman, Discrete groups of motions of spaces of constant curvature, from: “Geometry, II”, Encyclopaedia Math. Sci. 29, Springer, Berlin (1993) 139-248 MR1254933

[10] A Weil, On discrete subgroups of Lie groups. II, Ann. of Math. (2) 75 (1962) 578-602 MR0137793

Department of Mathematics, University of Pennsylvania David Rittenhouse Lab, 209 South 33rd Street, Philadephia, PA 19104-6395, USA

tarikb@sas.upenn.edu, peterastorm@gmail.com http://www.sas. upenn.edu/ pstorm/index.html

Received: 09 November 2008 